\title{
Optically induced magnetization in homogeneous, undoped dielectric media
}

\author{
S. C. Rand, W. M. Fisher, and S. L. Oliveira* \\ Division of Applied Physics, Randall Laboratory, University of Michigan, Ann Arbor, Michigan 48109-1022, USA \\ *Corresponding author: slo@ufgd.edu.br
}

Received October 12, 2007; revised March 19, 2008; accepted March 19, 2008;

posted March 27, 2008 (Doc. ID 88436); published June 5, 2008

\begin{abstract}
A widely held viewpoint in optics, namely, that dynamic magnetic effects are extremely weak at optical frequencies, is re-examined. Nonlinear charge motion induced by the optical magnetic field in dielectric systems is analyzed, is predicted to be resonantly enhanced, and is observed experimentally in $\mathrm{CCl}_{4}, \mathrm{C}_{6} \mathrm{H}_{6}$, and $\mathrm{H}_{2} \mathrm{O}$ at the fundamental input frequency. Excellent agreement is obtained with a classical magnetic harmonic oscillator model, which shows that the maximum dynamic magnetic dipole (MD) moment at optical frequencies is one half the electric dipole (ED) moment. As a consequence, magnetic dipole radiation generated by the optical magnetic field with an intensity one fourth that of ED radiation, as well as unanticipated nonlinear optical effects such as magnetic white-light generation, can arise in homogeneous transparent dielectrics. The mechanism of MD formation is confirmed experimentally to be second order in the input field, and the strength of the radiation is accounted for as a first-order contribution to the vector potential. Predictions are made of optical magnetic resonance, negative permeability, self-induced magnetic birefringence, and optically induced Faraday rotation. (C) 2008 Optical Society of America
\end{abstract}

OCIS codes: 190.0190, 190.4410, 190.7710, 320.7110, 350.3618.

\section{INTRODUCTION}

As is well-known, radiation fields can be calculated in a simple way from the motion of charges in a source region of interest using the vector potential $\bar{A}(\bar{r}, t)$. When decomposed into multipole contributions the source current in the leading term of $\bar{A}(\bar{r}, t)$ is taken to point along the incident electric field $\bar{E}(\bar{r}, t)$ and to give rise to an oscillating electric dipole (ED). This is because only the electric field can generate linear charge acceleration and, in the case of plane waves, the magnetic force on a charge $q$ traveling at velocity $v$ is $\bar{F}_{m}=q(\bar{v} \times \hat{k} \times \bar{E} / c)$ where $\hat{k}$ is the direction of propagation. This is smaller than the electric force by $v / c$, where $c$ is the velocity of light in vacuum. At nonrelativistic intensities $\left(I \ll 10^{18} \mathrm{~W} / \mathrm{cm}^{2}\right)$ scattered fields are therefore expected to be dominated by electric dipole radiation. In this paper it is nevertheless shown theoretically and experimentally that charge motion perpendicular to the electric field that is nonlinear and magnetic in origin can have important consequences at optical frequencies in transparent dielectrics at moderate intensities.

Historically the idea of producing magnetization by optical means dates back to the beginning of nonlinear optics. In 1961, Pitaevski [1], Shen and Bloembergen [2], and others [3] described theoretically how a static magnetization could be generated by an "effectively magnetic" interaction proportional to $\bar{E}(\omega) \times \bar{E}^{*}(-\omega)$ in the case of circularly polarized fields. Since that time, several groups have ascribed strong optically induced magnetization effects and ultrafast manipulation of static magnetizations with light to nonlinear, difference frequency interactions. For a recent review, the reader is referred to [4]. Addition- ally, when the optical intensity becomes high enough to accelerate electrons to nearly the speed of light during an optical cycle it is well-known that magnetic interactions become important [5]. Here, however, we describe dynamic magnetization that is generated through the action of the optical magnetic field $\bar{B}(\omega)$ via a nonlinear interaction proportional to the tensor product $\bar{E}(\omega) \bar{B}(\omega)$ at nonrelativistic intensities. Despite its dependence on two input fields (neither conjugate nor circular) this optically induced magnetization will presently be shown to be intense and to oscillate at the fundamental frequency $\omega$ rather than at a difference or harmonic frequency.

The initial terms of the multipole expansion for the vector electromagnetic potential produced in the far field by an arbitrary current distribution specified by $\bar{J}\left(\bar{r}^{\prime}\right)$ can be written [6] as

$$
\begin{aligned}
\frac{4 \pi}{\mu_{0}} \bar{A}(\bar{r}, t)= & \frac{1}{R} \int \bar{J}\left(\bar{r}^{\prime}, t^{\prime}\right) \mathrm{d}^{3} r^{\prime} \\
& -\left[\frac{\partial}{\partial x_{\alpha}}\left(\frac{1}{r}\right)\right]_{R} \int r_{\alpha}^{\prime} \bar{J}\left(\bar{r}^{\prime}, t^{\prime}\right) \mathrm{d}^{3} r^{\prime}+\cdots .
\end{aligned}
$$

Here the position vector of the field point is $\bar{R}$ and that of the source is $\bar{r}^{\prime}$; so the vector potential depends on $\bar{r}=\bar{R}$ $-\bar{r}^{\prime}$, the magnitude of which is just $R$ in the far field, with a retardation of $t^{\prime}=t-R / c$. The second term is separable into symmetric and antisymmetric parts that correspond to magnetic dipole and electric quadrupole components, respectively. Following this decomposition the ratio of the magnetic dipole moment $m$ (from the second term) to the 
ED moment $p$ (from the first term) is found to be very small for source regions the size of atoms or molecules at optical frequencies. In the experiments reported here on molecular liquids the dipole approximation $(2 \pi a / \lambda \ll 1)$ is certainly upheld and so one can estimate the ratio (setting angular momentum of the charge to be Planck's constant $\hbar)$ to be

$$
\left|\frac{m}{p}\right|=\left(\frac{\hbar / m_{e} c}{a_{0}}\right) \ll 1,
$$

where $m_{e}$ is the electron rest mass and $a_{0}$ is the Bohr radius. The ratio in Eq. (1.2) is equal to the fine structure constant $\alpha \equiv e^{2} / \hbar c \cong 1 / 137$ and the relative intensity of the magnetic dipole radiation is proportional to its square. Since $\alpha^{2}$ is extremely small, magnetic dipole radiation originating from the second term in Eq. (1.1) is ignored in optical interactions. Landau and Lifshitz [7] stated that "there is no meaning in using magnetic susceptibility from optical frequencies onward, and in discussing such phenomena we must put $\mu=1$." However this traditional viewpoint overlooks the possibility of a large oscillatory magnetic dipole moment in the first term of Eq. (1.1), originating from a nonlinear source current.

Because the current density in the first term of Eq. (1.1) is induced by forces that cause linear and circular motion, it can be decomposed into linear (electric) and circular (magnetic) components, $\bar{J}_{E, \omega}$ and $\bar{J}_{M, \omega}$, respectively, to obtain

$$
\begin{aligned}
\bar{A}_{\omega}(\bar{r})= & \frac{\mu_{0} e^{-i k r}}{4 \pi r} \int \bar{J}_{E, \omega}\left(\bar{r}^{\prime}\right) \mathrm{d}^{3} r^{\prime}+\frac{\mu_{0} e^{-i k r}}{4 \pi r} \int \bar{J}_{M, \omega}\left(\bar{r}^{\prime}\right) \mathrm{d}^{3} r^{\prime} \\
& +\cdots
\end{aligned}
$$

In Eq. (1.3) the subscript $\omega$ indicates that an harmonic approximation has been made for the time dependence of $\bar{A}(\bar{r}, t) . \bar{J}_{E, \omega}$ and $\bar{J}_{M, \omega}$ are distinguished by different continuity relations $\bar{\nabla} \cdot \bar{J}_{E, \omega}=-\partial \rho / \partial t$ and $\bar{\nabla} \cdot \bar{J}_{M, \omega}=0 . \bar{J}_{M}$ oscillates around the magnetic field at frequency $\omega$ in the plane formed by the electric field and the wave vector of the light. It therefore contains a component perpendicular to $\bar{E}$ and parallel to wave vector $\bar{k}$.

In Eq. (1.3) the first term on the right containing $\bar{J}_{E, \omega}$ yields an ED. The second one containing $\bar{J}_{M, \omega}$ can be shown to be strictly magnetic dipole (MD). This result is given in Appendix A. Currents of the $\bar{J}_{M, \omega}$ type are generated by magnetic Lorentz forces in all matter, but are usually negligible in weak optical interactions. As we shall show, however, the motion perpendicular to $\bar{E}$ can be resonantly enhanced in bound electron systems at moderate intensities, resulting in magnetic dipole strengths comparable to those of electric dipoles in the same system. In this way, magnetic effects ordinarily reserved for the relativistic regime [5] become observable at much lower intensities. Moreover it can be anticipated that in dielectric media large ensembles of optical MDs of this type will not suffer from diamagnetic cancellation. Because the optically driven charge motion of bound electrons does not form closed current loops (at nonrelativistic intensities), no opposing current cancellation can take place between neighboring molecules. The macroscopic magnetic response will be additive and large like electric polarization.

In Section 2 a model is developed for simple harmonic radial and torsional motion of bound charges interacting with light. The treatment is restricted to linear restoring forces (or a parabolic intramolecular potential) to emphasize that none of the results described in this paper are related to nonlinear electric field effects-proportional to $E^{2}, E^{3}$, etc. Such terms, mediated by nonparabolicity of the intramolecular potential in nonlinear optics, are excluded from the model. It is shown that a magnetic moment proportional to the incident light intensity can arise and grow to a maximum value of one half the ED moment. That is, the magnetic scattering intensity is predicted to be quadratic with respect to input intensity below saturation $\left(I<I_{s}\right)$, and to maintain a constant proportionality with respect to the electric polarization over a wide range above it. In the saturation regime $\left(I>I_{s}\right)$, this phenomenon mimics a magnetic scattering process that is linear rather than nonlinear in the intensity. The predicted polarization, frequency, intensity dependence, and saturated relative intensity of the magnetic scattering are all shown to be in excellent accord with experimental observations in several dielectric liquids. This work therefore has numerous interesting consequences that were not realized in the early days of nonlinear optics.

\section{CLASSICAL THEORETICAL MODEL}

Classical continuum theory accurately describes optical interactions far from electronic resonances. Hence the standard model that explains optical absorption, dispersion, and resonance in bound electron systems based on Newton's second law of motion [8] is quantitatively precise in regions of transparency. An important element of this model is that a restoring force counterbalances the driving force exerted on charges by the electromagnetic fields. In the simplest case the restoring force is linear in the displacement of electrons (charge $-e$ and mass $m_{e}$ ) from equilibrium positions. Here we make the same assumption, but extend the idea of a radiant polarization driven by electric displacement currents to magnetization driven by magnetic displacement currents. Magnetic forces are included in the equation of motion and the restoring forces are not assumed to be purely radial, but may have different components parallel and perpendicular to the main driving field $\bar{E}$.

Consider a plane electromagnetic wave that is polarized along $\hat{x}$ and propagates along the $\hat{z}$ axis through a centrosymmetric system of charges initially at rest, as shown in Fig. 1. The assumption of centrosymmetry is not necessary, but is helpful in interpreting the analytic results and experiments in centrosymmetric samples. Electric and magnetic field components may be represented by 


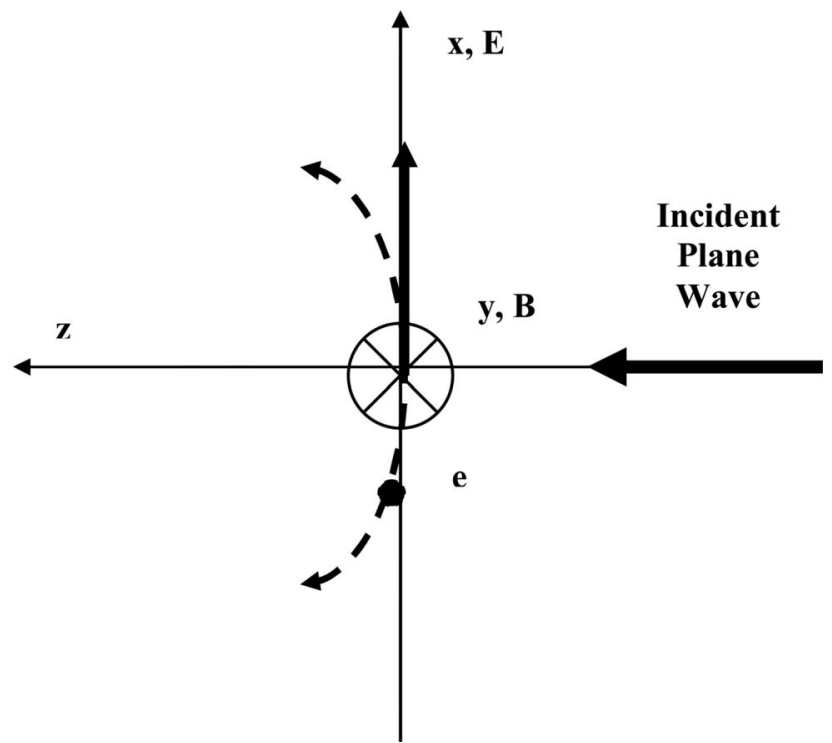

Fig. 1. Classical charge motion produced by an electromagnetic wave polarized along $x$ and propagating along $z$. Dashed (solid) vertical arrows schematically indicate motion with (without) the Lorentz force.

$$
\begin{aligned}
& \bar{E}(z, t)=\frac{1}{2} \hat{x}\left(E_{0} \exp [-i(\omega t-k z)]+\text { c.c. }\right), \\
& \bar{B}(z, t)=\frac{1}{2} \hat{y}\left(B_{0} \exp [-i(\omega t-k z)]+\text { c.c. }\right) .
\end{aligned}
$$

Allowing for charge displacements in both the $\hat{x}$ and $\hat{z}$ directions (due to $\bar{E}$ and $\bar{B}$, respectively), the equation of motion $m_{e}\left(\partial^{2} \bar{r}^{\prime} / \partial t^{2}\right)=\bar{F}$ in component form yields

$$
\begin{gathered}
\frac{\partial^{2} x(t)}{\partial t^{2}}+\gamma_{1} \frac{\partial x(t)}{\partial t}+\omega_{1}^{2} x(t)=-\frac{e E(t)}{m_{e}}+\frac{e B(t)}{m_{e}} \frac{\partial z(t)}{\partial t}, \\
\frac{\partial^{2} z(t)}{\partial t^{2}}+\gamma_{2} \frac{\partial z(t)}{\partial t}+\omega_{2}^{2} z(t)=-\frac{e B(t)}{m_{e}} \frac{\partial x(t)}{\partial t} .
\end{gathered}
$$

Since restoring forces parallel and perpendicular to $\bar{E}$ are independent, the corresponding force constants $K_{1}$ $\equiv m_{e} \omega_{1}^{2}$ and $K_{2} \equiv m_{e} \omega_{2}^{2}$ for motion along $\hat{x}$ and $\hat{z}$, respectively, are assumed to be unequal $\left(K_{1} \neq K_{2}\right)$. Orthogonal motions of electric charges in the intramolecular potential well sample different topography of the potential surface. This is illustrated in Fig. 2, where the slope of the potential surface can be seen to differ for radial versus azimuthal motion of a bound charge. Similarly, the damping coefficients $\gamma_{1}$ and $\gamma_{2}$ are assumed to be unequal $\left(\gamma_{1}\right.$ $\left.\neq \gamma_{2}\right)$.

Taking the optical fields $\bar{E}$ and $\bar{B}$ to be in phase, as they are in vacuum and dilute media, nontransient solutions to Eqs. (2.1) and (2.2) that describe charge motion have the simple forms

$$
\begin{gathered}
x(t)=\frac{1}{2}\left[x_{0} \exp (-i \omega t)+x_{0}^{*} \exp (i \omega t)\right], \\
z(t)=\frac{1}{2}\left[z_{0} \exp (-i 2 \omega t)+z_{0}^{*} \exp (i 2 \omega t)\right] .
\end{gathered}
$$

Solutions for the displacements $x(t)$ and $z(t)$ are needed to determine the magnitudes of the current densities $J_{E}$

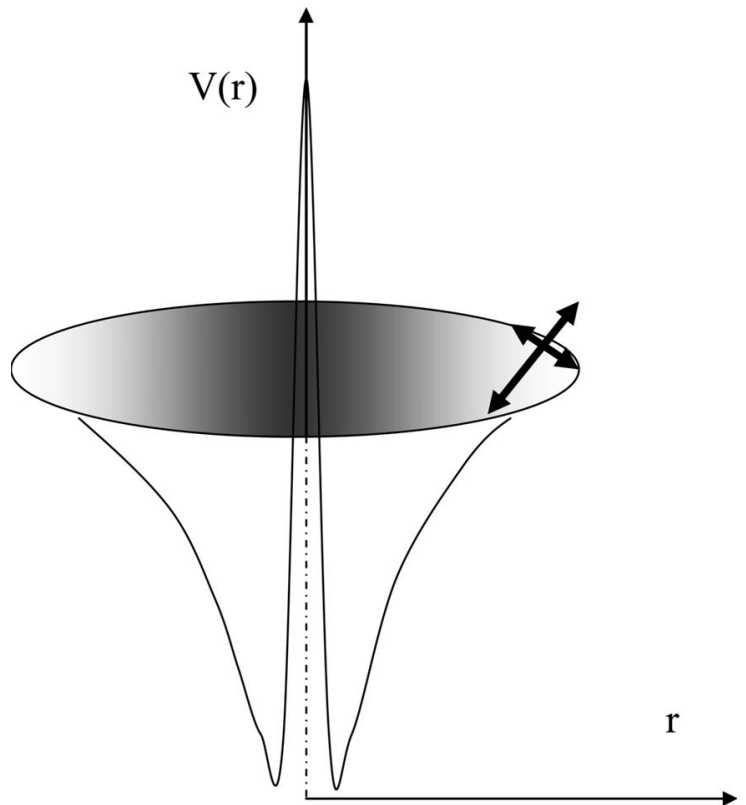

Fig. 2. Illustration of the dependence of restoring forces on direction of classical motion of a charge in a simple molecular potential well $V(r)$. The slopes of the potential (and therefore the restoring forces) are different for motions in the two directions indicated by double-headed arrows.

$=-N e \dot{x}$ and $J_{M}=-2 N e \dot{z}$. Solving for the amplitudes by substituting Eqs. (2.3) and (2.4) into (2.1) and (2.2), we find

$$
\begin{gathered}
x_{0}=\frac{\left(e E_{0} / m_{e}\right)}{\left(\omega^{2}+i \omega \gamma_{1}-\omega_{1}^{2}\right)\left(1+N\left(\omega, B_{0}\right)\right)} \\
z_{0}=\frac{1}{2} \frac{-i \omega \omega_{c}\left(e E_{0} / m_{e}\right)}{\left(\omega^{2}+i \omega \gamma_{1}-\omega_{1}^{2}\right)\left(4 \omega^{2}+2 i \omega \gamma_{2}-\omega_{2}^{2}\right)\left(1+N\left(\omega, B_{0}\right)\right)} .
\end{gathered}
$$

The magnetic renormalization factor $N\left(\omega, B_{0}\right)$ in Eqs. (2.5) and (2.6) is given by

$$
N\left(\omega, B_{0}\right)=\omega^{2} \omega_{c}^{2} / 2\left(\omega^{2}+i \omega \gamma_{1}-\omega_{1}^{2}\right)\left(4 \omega^{2}+2 i \omega \gamma_{1}-\omega_{2}^{2}\right),
$$

where $\omega_{c} \equiv e B_{0} / m_{e}$ is the cyclotron frequency.

Notice that the charge trajectories produce oscillatory motion along both the $\hat{x}$ and $\hat{z}$ directions, as indicated in Fig. 1. Charge motion along the direction of propagation is a projection along $z$ of the equivalent magnetic current $\bar{J}_{M}(t)$ that oscillates at the fundamental frequency $\omega$ as electrons curl around the $\bar{B}$ field. It is important to note that this motion resolves itself into a doubled oscillation along the fixed Cartesian $z$ axis, as assumed in Eq. (2.4). Both the $z$-directed oscillation at $2 \omega$, and its phase shift by $\pi / 2$ with respect to motion along the $x$ axis, can easily be pictured by inspection of the figure. As the charge moves from $-x_{0}$ to $+x_{0}$ and back to $-x_{0}$, completing one cycle of the electric polarization, the motion along $z$ undergoes two cycles centered about a displaced origin. Since the restoring force in our model is strictly linear, this motion at $2 \omega$ is the result of the (nonlinear) magnetic 
force and can arise even in centrosymmetric media where quadratic restoring forces are absent and harmonic generation by the electric field alone cannot take place. The frequency-doubled solution for $z$-directed motion yields a current density $\bar{J}_{M}$ that circulates around $\bar{B}$ at frequency $\omega$. To show this explicitly, the magnetic current $\left[\bar{J}_{M}(\omega)\right]_{s p h}=J_{M} \hat{\theta} \cos \omega t$ written in spherical coordinates can be transformed to Cartesian coordinates using the substitution $\hat{\theta}=\hat{x} \cos \omega t-\hat{z} \sin \omega t$. This yields

$$
\begin{aligned}
{\left[\bar{J}_{M}(2 \omega)\right]_{\text {Cart }} } & =J_{M}[\hat{x} \cos \omega t-\hat{z} \sin \omega t] \cos \omega t \\
& =\frac{1}{2} J_{M}[\hat{x}(1+\cos 2 \omega t)-\hat{z} \sin 2 \omega t] .
\end{aligned}
$$

Thus, projection of the axial vector $\left[\bar{J}_{M}(\omega)\right]_{s p h}$ onto fixed Cartesian axes produces two vector current components that are of polar character, doubles the frequency of motion, halves the amplitude, and shifts the origin of charge motion. This shows that $\left[\bar{J}_{M}(\omega)\right]_{s p h}$ yields a MD oriented parallel to $\bar{B}$, oscillating at frequency $\omega$ following the prescription $\bar{J}_{M}(\omega)=\bar{\nabla} \times \bar{M}(\omega)$. However, in Cartesian coordinates, the polarization responsible for MD emission at the fundamental frequency appears frequency-doubled, in a form that might be written as $P_{z}(2 \omega)=-N e z_{0}(2 \omega)$, since it is proportional to the projection of $\bar{J}_{M}(\omega)$ on $z$. Note that in the present model this polarization can only generate magnetic dipole radiation, since second-harmonic electric dipole radiation is forbidden by centrosymmetry. Moreover, $\bar{J}_{E}(\omega)$ obeys the charge conservation equation $\bar{\nabla} \cdot \bar{J}_{E}$ $=-\partial \rho / \partial t$ for electric currents, whereas $\bar{J}_{M}(\omega)$ does not $\left(\bar{\nabla} \cdot \bar{J}_{M}=0\right)$.

The frequencies of electric and magnetic resonance are found by setting the denominators in Eqs. (2.5) and (2.6) equal to zero. There is a common resonance at $\omega=\omega_{1}$, but motion along $z$ also exhibits a second resonance at

$$
\omega=\omega_{2} / 2 .
$$

This purely magnetic resonance condition calls for an optical frequency that is half the resonant frequency of linear motion along $z$. This tuning requirement is significantly relaxed at high intensities, as described in a forthcoming publication [9].

The intensity-dependent ratio of magnetic to electric field displacement current densities may be evaluated directly from Eqs. (2.5) and (2.6). Far from ED resonance, this yields

$$
R=\left|J_{M} / J_{E}\right|=|2 \dot{z} / \dot{x}|=\frac{2 \omega \omega_{c}\left[\left(4 \omega^{2}-\omega_{2}^{2}\right)^{2}+\left(2 \omega \gamma_{2}\right)^{2}\right]^{1 / 2}}{\left(4 \omega^{2}-\omega_{2}^{2}\right)^{2}+4 \omega^{2} \gamma_{2}^{2}} .
$$

$R$ depends linearly on the input field amplitude through its proportionality to $\omega_{c}$ and hence to $B$. Consequently the ratio of magnetic to electric emission intensity $\left(\sim R^{2}\right)$ is predicted to rise linearly with optical intensity and to exhibit purely magnetic resonant enhancement. On resonance, $R$ becomes of order unity as $\omega_{c} \rightarrow \gamma_{2}$.

To determine the maximum ratio $R_{\max }$ of magnetic to electric current densities at elevated power levels, we now integrate Ampère's law using the geometry of Fig. 3 for a

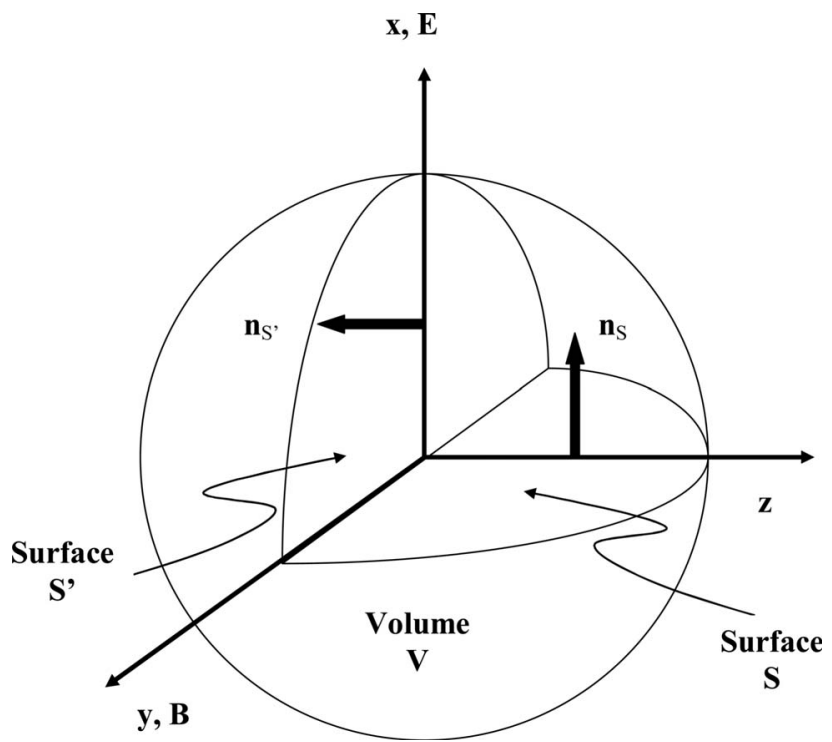

Fig. 3. Geometry for integration of Ampère's law to determine relative magnitudes and phases of electric and magnetic current density.

plane wave propagating along the $z$ axis with its electric field linearly polarized along $\hat{x}$ and its magnetic field oriented along $\hat{y}$. The wave impinges on an arbitrary spherical volume $V$ containing a uniform density $N$ of bound electrons. These charges establish a polarization current density $\bar{J}_{p}=\dot{\bar{P}}$ and a magnetic displacement current $\bar{J}_{M}$ according to

$$
\bar{\nabla} \times \bar{H}=\varepsilon_{0} \dot{\bar{E}}+\bar{J}_{p}+\bar{J}_{M} .
$$

Allowing for current components parallel and perpendicular to $\bar{E}$, we write

$$
\bar{J}_{p}=\bar{J}_{p, \perp}+\bar{J}_{p, \|}, \quad \bar{J}_{M}=\bar{J}_{M, \perp}+\bar{J}_{M, \|},
$$

where $\bar{J}_{M, \perp}=-J_{M} \hat{z} \sin \omega t$ and $\bar{J}_{M, \|}=J_{M} \hat{x} \cos \omega t$ as in Eq. (2.8). Next we substitute Eq. (2.12) into Eq. (2.11). Ampère's law can then be integrated over surface $S$ in Fig. 3. Components perpendicular to $\bar{E}$ are orthogonal to the surface normal $\hat{n}_{S}$. Hence the result is

$$
\int_{S}(\bar{\nabla} \times \bar{H}) \cdot \mathrm{d} \bar{s}=\varepsilon_{0} \int_{S} \dot{\bar{E}} \cdot \mathrm{d} \bar{s}+\int_{S} \bar{J}_{p, \|} \cdot \mathrm{d} \bar{s}+\int_{S} \bar{J}_{M, \|} \cdot \mathrm{d} \bar{s} .
$$

To deduce a relationship between optically induced currents $\bar{J}_{p}$ and $\bar{J}_{M}$, one can specialize the calculation to optical frequencies by substituting Faraday's law $(\bar{\nabla}$ $\times \bar{E}) / i \omega \mu$ for $\bar{H}$ in Eq. (2.13). Furthermore we set $\mu$ $=\mu_{0}\left(1+\chi_{m}\right)$, and consider $\chi_{m} \ll 1$, which ostensibly limits the estimate of $R_{\max }$ to transparent media with small polarization and magnetization far from resonance. While our experiments were indeed performed in this limit, the theoretical result obtained for $R_{\text {max }}$ below is more general, as will become evident.

With these approximations, the integral on the left side of Eq. (2.13) is equal to the first term on the right. Hence one obtains 


$$
0=\int_{S} \bar{J}_{p, \|} \cdot \mathrm{d} \bar{s}+\int_{S} \bar{J}_{M, \|} \cdot \mathrm{d} \bar{s},
$$

or

$$
\int_{S} \bar{J}_{M, \|} \cdot \mathrm{d} \bar{s}=-\int_{S} \bar{J}_{p, \|} \cdot \mathrm{d} \bar{s} .
$$

A similar integration over surface $S^{\prime}$ in Fig. 3 yields

$$
\int_{S^{\prime}} \bar{J}_{M, \perp} \cdot \mathrm{d} \bar{s}=-\int_{S^{\prime}} \bar{J}_{p, \perp} \cdot \mathrm{d} \bar{s} .
$$

Because the amplitudes of $\bar{J}_{M}$ parallel and perpendicular to the electric field are equal $\left(J_{M, \perp}=J_{M, \|}=J_{M}\right)$, the addition of Eqs. (2.15) and (2.16) yields the result

$$
J_{M}=-\frac{1}{2}\left[J_{p}\right]_{\mathrm{tot}}=-\frac{1}{2} J_{E} .
$$

Since the input electric and magnetic fields are in phase, the sign in Eq. (2.17) is a phase factor indicating that the magnetic moment opposes the optical magnetic field. The ratio $R_{\max }=\left|J_{M} / J_{E}\right|=1 / 2$ calculated in Eq. (2.17) is the same as the ratio of magnetic to electric dipole moments of a perfectly conducting sphere in magnetostatics [10], but here it is obtained in a classical model that treats bound electrons as the carriers of electric and magnetic displacement current densities at optical frequencies. It indicates that of all the charges displaced along $x$ by $\bar{E}$, at most half can turn in the $\bar{B}$ field and contribute to positive magnetic current by passing through the surface $S^{\prime}$.

Scattered electromagnetic fields in the radiation zone may be calculated using the expressions [11]

$$
\begin{gathered}
\bar{E}_{\mathrm{rad}}=\frac{1}{4 \pi \varepsilon_{0} c^{2}} \int \frac{([\dot{\bar{J}}] \times \hat{r}) \times \hat{r}}{r} \mathrm{~d} V, \\
\bar{H}_{\mathrm{rad}}=\frac{1}{4 \pi c} \int \frac{[\dot{\bar{J}}] \times \hat{r}}{r} \mathrm{~d} V,
\end{gathered}
$$

where square brackets indicate evaluation at the retarded time and the integration is performed over source volume $V$. Dots indicate time derivatives and $\hat{r}$ is a unit vector in the direction of the point of observation, at a distance $r$ from the scattering volume. Knowledge of the vector current densities $\bar{J}_{E}$ and $\bar{J}_{M}$ (assumed to be uniform in this continuum model) associated with time-varying ED and MD moments is enough to determine the electric and magnetic dipole components of light radiated from the sample. This can be seen explicitly by calculating the ratio of the magnitudes of the Poynting vector $\bar{S}=\bar{E}_{\text {rad }}$ $\times \bar{H}_{\text {rad }}$ for electric and magnetic radiation, namely $S_{M} / S_{E}$, given by

$$
\begin{aligned}
\frac{S_{M}}{S_{E}} & =\left|\int \frac{\omega\left(\bar{J}_{M} \times \hat{r}\right) \times \hat{r} \mathrm{~d} V^{\prime}}{r} \times \int \frac{\omega\left(\bar{J}_{M} \times \hat{r}\right) \mathrm{d} V^{\prime}}{r}\right| /\left|\int \frac{\omega\left(\bar{J}_{E} \times \hat{r}\right) \times \hat{r} \mathrm{~d} V^{\prime}}{r} \times \int \frac{\omega\left(\bar{J}_{E} \times \hat{r}\right) \mathrm{d} V^{\prime}}{r}\right| \\
& =\frac{J_{M}^{2}}{J_{E}^{2}}\left|\int \frac{\left(\hat{J}_{M} \times \hat{r}\right) \times \hat{r} \mathrm{~d} V^{\prime}}{r} \times \int \frac{\hat{J}_{M} \times \hat{r} \mathrm{~d} V^{\prime}}{r}\right| /\left|\int \frac{\left(\hat{J}_{E} \times \hat{r}\right) \times \hat{r} \mathrm{~d} V^{\prime}}{r} \times \int \frac{\hat{J}_{E} \times \hat{r} \mathrm{~d} V^{\prime}}{r}\right|=\frac{J_{M}^{2}}{J_{E}^{2}} .
\end{aligned}
$$

Since the maximum magnetic current density is $\left[J_{M}\right]_{\max }$ $=R_{\text {max }} J_{E}=(1 / 2) J_{E}$, it follows that the ratio of far-field intensities cannot exceed

$$
\frac{S_{M}}{S_{E}}=R_{\max }^{2}=\frac{1}{4} .
$$

Equation (2.21) gives the maximum possible value of the ratio $R$ of magnetic dipole to electric dipole emission intensity in dielectric materials. Based on Eq. (2.10), this ratio depends not only on intensity but also on the material, since the magnetic resonant denominator must play an important role in accounting quantitatively for system response. A complete treatment of the dynamics, addressing aspects of primary parametric resonance at high modulation index [12], is deferred to another paper [9] however.

Equation (2.21) shows that with full resonant enhancement, the dynamic magnetic dipole moment due to the passage of light in the nonrelativistic limit may be one half the electric dipole moment. The argument of Appendix A explains how such a magnetic dipole contributes to the first multipole term of the vector potential, yielding MD radiation one fourth the intensity of ED emission and transcending the traditional limitations on magnetic effects described in Section 1. In the experimental portion of this paper, we show that intense magnetic effects are indeed observable at optical frequencies, bearing out these expectations.

\section{ELECTRIC AND MAGNETIC SUSCEPTIBILITIES}

We now solve for the gyrotropic response of an anisotropic system subjected to a transverse electromagnetic plane wave. The fields $\bar{E}(t)$ and $\bar{B}(t)$, as well as the charge displacement from equilibrium $\bar{r}^{\prime}(t)$, are assumed to vary harmonically in time. The wave is assumed to propagate along the $z$ axis so that Cartesian components of the fields and displacement are given by 


$$
\begin{gathered}
\bar{E}=\frac{1}{2}\left[E_{0 x} \hat{x}+E_{0 y} \hat{y}\right] e^{-i \omega t}+\text { c.c. } \\
\bar{B}=\frac{1}{2}\left[B_{0 x} \hat{x}+B_{0 y} \hat{y}\right] e^{-i \omega t}+\text { c.c. } \\
\bar{r}^{\prime}=\left[x_{0}^{(0)} \hat{x}+y_{0}^{(0)} \hat{y}+z_{0}^{(0)} \hat{z}\right]+\frac{1}{2}\left[x_{0}^{(1)} \hat{x}+y_{0}^{(1)} \hat{y}+z_{0}^{(1)} \hat{z}\right] e^{-i \omega t}+c . c . \\
+\frac{1}{2}\left[x_{0}^{(2)} \hat{x}+y_{0}^{(2)} \hat{y}+z_{0}^{(2)} \hat{z}\right] e^{-2 i \omega t}+\text { c.c. },
\end{gathered}
$$

respectively. The equation of motion can then be written as

$$
m_{e} \ddot{\bar{r}}^{\prime}+m_{e} \dot{\bar{r}} \dot{\bar{r}}^{\prime}+K \bar{r}^{\prime}=-e \bar{E}-e \overline{\dot{r}} \times \bar{B},
$$

where $-m_{e} \dot{\bar{r}}^{\prime}$ provides viscous damping and $-K \bar{r}^{\prime}$ is the restoring force. We shall assume that both the damping constant $\gamma$ and the spring constant $K$ may be different in different directions.

By identifying the electric polarization $\bar{P}(t)=-N e \bar{r}^{\prime}(t)$, and its frequency components according to $\bar{P}(t)=P^{(0)}$ $+\frac{1}{2}\left[P^{(1)} e^{-i \omega t}+P^{(1)^{*}} e^{i \omega t}\right]+\frac{1}{2}\left[P^{(2)} e^{-i 2 \omega t}+P^{(2)^{*}} e^{i 2 \omega t}\right], \quad$ coupled equations for all the terms of $P$ can be obtained directly from Eq. (3.1). The starting equations for Cartesian components of $\bar{P}(t)$ are

$$
\begin{gathered}
m_{e} \ddot{P}_{x}+m_{e} \gamma_{x} \dot{P}_{x}+K_{x} P_{x}=N e^{2} E_{0 x}-e\left(\dot{P}_{y} B_{0 z}-\dot{P}_{z} B_{0 y}\right), \\
m_{e} \ddot{P}_{y}+m_{e} \gamma_{y} \dot{P}_{y}+K_{y} P_{y}=N e^{2} E_{0 y}-e\left(\dot{P}_{z} B_{0 x}-\dot{P}_{x} B_{0 z}\right), \\
m_{e} \ddot{P}_{z}+m_{e} \gamma_{z} \dot{P}_{z}+K_{z} P_{z}=-e\left(\dot{P}_{x} B_{0 y}-\dot{P}_{y} B_{0 x}\right) .
\end{gathered}
$$

System susceptibilities that account for magnetization effects may be calculated by solving Eqs. (3.2)-(3.4) for the components of $P_{x}, P_{y}$, and $P_{z}$ at frequencies appearing in the expansion of $\bar{r}^{\prime}(t)$ given above, namely $0, \omega$, and $2 \omega$. Specifically, $\chi$ is evaluated according to the relations

$$
\begin{gathered}
{[\chi(0)]_{i j}=P_{i}^{(0)} / \varepsilon_{0} E_{0 j},} \\
{[\chi(\omega)]_{i j}=P_{i}^{(1)} / \varepsilon_{0} E_{0 j},} \\
{[\chi(2 \omega)]_{i j}=P_{i}^{(2)} / \varepsilon_{0} E_{0 j},}
\end{gathered}
$$

where $i, j$, and $k$ are Cartesian coordinates $x, y$, or $z$. When defined in this way, the susceptibilities are all dimensionless, and have the merit of showing the nonlinear magnetic contributions explicitly. However different orders of the nonlinear interactions appear together in the susceptibility at each frequency.

If we retain only the leading terms in the susceptibilities (written in Cartesian coordinates), that is only those terms depending on one or two input magnetic field amplitudes, the results for nonzero contributions to the susceptibility $\chi$ are as follows.

Zero frequency (0):

$$
[\chi(0 ; \omega,-\omega)]_{12}=\frac{N e^{2}}{\varepsilon_{0}}\left[\frac{i e \omega B_{0 z}^{*}}{4 K_{x} \Delta_{y} F_{y} G_{y}}+\text { c.c. }\right],
$$

$$
\begin{gathered}
{[\chi(0 ; \omega,-\omega)]_{21}=\frac{N e^{2}}{\varepsilon_{0}}\left[\frac{-i e \omega B_{0 z}^{*}}{4 K_{y} \Delta_{x} F_{x}}+\text { c.c. }\right],} \\
{[\chi(0 ; \omega,-\omega)]_{13}=\frac{N e^{2}}{\varepsilon_{0}}\left[\frac{-i e \omega B_{0 y}^{*}}{4 K_{x} \Delta_{z} F_{z} G_{z}}+\text { c.c. }\right],} \\
{[\chi(0 ; \omega,-\omega)]_{31}=\frac{N e^{2}}{\varepsilon_{0}}\left[\frac{i e \omega B_{0 y}^{*}}{4 K_{z} \Delta_{x} F_{x}}+\text { c.c. }\right],} \\
{[\chi(0 ; \omega,-\omega)]_{23}=\frac{N e^{2}}{\varepsilon_{0}}\left[\frac{i e \omega B_{0 x}^{*}}{4 K_{y} \Delta_{z} F_{z} G_{z}}+\text { c.c. }\right],} \\
{[\chi(0 ; \omega,-\omega)]_{32}=\frac{N e^{2}}{\varepsilon_{0}}\left[\frac{-i e \omega B_{0 x}^{*}}{4 K_{z} \Delta_{y} F_{y} G_{y}}+\text { c.c. }\right] .}
\end{gathered}
$$

Fundamental frequency $(\omega)$ :

$$
[\chi(\omega ; \omega)]_{11}=\frac{N e^{2}}{\varepsilon_{0}}\left[\frac{1}{\Delta_{x} F_{x}}\right],
$$

$$
[\chi(\omega ; \omega,-\omega, \omega)]_{12}=\frac{N e^{2}}{\varepsilon_{0}}\left[\frac{-e^{2} \omega^{2} B_{0 x} B_{0 y}^{*}}{2 \Delta_{x} \Delta_{y} \Delta_{z}^{\prime} F_{x} F_{y} G_{y}}\right],
$$

$$
[\chi(\omega ; \omega,-\omega, \omega)]_{21}=\frac{N e^{2}}{\varepsilon_{0}}\left[\frac{-e^{2} \omega^{2} B_{0 x}^{*} B_{0 y}}{2 \Delta_{x} \Delta_{y} \Delta_{z}^{\prime} F_{x} F_{y} G_{y}}\right],
$$

$$
[\chi(\omega ; \omega)]_{22}=\frac{N e^{2}}{\varepsilon_{0}}\left[\frac{1}{\Delta_{y} F_{y} G_{y}}\right],
$$

$$
[\chi(\omega ; \omega,-\omega, \omega)]_{23}=\frac{N e^{2}}{\varepsilon_{0}}\left[\frac{-e^{2} \omega^{2} B_{0 y} B_{0 z}^{*}}{2 \Delta_{x}^{\prime} \Delta_{y} \Delta_{z} F_{y} F_{z} G_{y} G_{z}}\right],
$$

$$
[\chi(\omega ; \omega,-\omega, \omega)]_{32}=\frac{N e^{2}}{\varepsilon_{0}}\left[\frac{-e^{2} \omega^{2} B_{0 y}^{*} B_{0 z}}{2 \Delta_{x}^{\prime} \Delta_{y} \Delta_{z} F_{y} F_{z} G_{y} G_{z}}\right],
$$

$$
\begin{gathered}
{[\chi(\omega ; \omega)]_{33}=\frac{N e^{2}}{\varepsilon_{0}}\left[\frac{1}{\Delta_{z} F_{z} G_{z}}\right],} \\
{[\chi(\omega ; \omega,-\omega, \omega)]_{31}=\frac{N e^{2}}{\varepsilon_{0}}\left[\frac{-e^{2} \omega^{2} B_{0 x}^{*} B_{0 z}}{2 \Delta_{x} \Delta_{y}^{\prime} \Delta_{z} F_{x} F_{z} G_{z}}\right],} \\
{[\chi(\omega ; \omega, \omega,-\omega)]_{13}=\frac{N e^{2}}{\varepsilon_{0}}\left[\frac{-e^{2} \omega^{2} B_{0 x} B_{0 z}^{*}}{2 \Delta_{x} \Delta_{y}^{\prime} \Delta_{z} F_{x} F_{z} G_{z}}\right] .}
\end{gathered}
$$

Second-harmonic frequency $(-2 \omega)$ :

$$
[\chi(2 \omega ; \omega, \omega)]_{12}=\frac{N e^{2}}{\varepsilon_{0}}\left[\frac{i e \omega B_{0 z}}{2 \Delta_{x}^{\prime} \Delta_{y} F_{y} G_{y}}\right],
$$




$$
\begin{aligned}
{[\chi(2 \omega ; \omega, \omega)]_{21} } & =\frac{N e^{2}}{\varepsilon_{0}}\left[\frac{-i e \omega B_{0 z}}{2 \Delta_{x} \Delta_{y}^{\prime} F_{x}}\right], \\
{[\chi(2 \omega ; \omega, \omega)]_{13} } & =\frac{N e^{2}}{\varepsilon_{0}}\left[\frac{-i e \omega B_{0 y}}{2 \Delta_{x}^{\prime} \Delta_{z} F_{z} G_{z}}\right], \\
{[\chi(2 \omega ; \omega, \omega)]_{31} } & =\frac{N e^{2}}{\varepsilon_{0}}\left[\frac{i e \omega B_{0 y}}{2 \Delta_{x} \Delta_{z}^{\prime} F_{x}}\right], \\
{[\chi(2 \omega ; \omega, \omega)]_{32} } & =\frac{N e^{2}}{\varepsilon_{0}}\left[\frac{-i e \omega B_{0 x}}{2 \Delta_{y} \Delta_{z}^{\prime} F_{y} G_{y}}\right], \\
{[\chi(2 \omega ; \omega, \omega)]_{23} } & =\frac{N e^{2}}{\varepsilon_{0}}\left[\frac{i e \omega B_{0 x}}{2 \Delta_{y}^{\prime} \Delta_{z} F_{z} G_{z}}\right] .
\end{aligned}
$$

In the expressions above, the resonant denominators are defined in terms of Cartesian coordinates $i=x, y, z$ to be

$$
\begin{gathered}
\Delta_{i} \equiv-m_{e} \omega^{2}-i m_{e} \gamma_{i} \omega+K_{i}, \\
\Delta_{i}^{\prime} \equiv-4 m_{e} \omega^{2}-2 i m_{e} \gamma_{i} \omega+K_{i} .
\end{gathered}
$$

The various $F$ factors can be written using cyclic permutation of the indices as

$$
F_{i} \equiv 1-\frac{e^{2} \omega^{2}}{2 \Delta_{i}}\left(\frac{\left|B_{0 k}\right|^{2}}{\Delta_{j}^{\prime}}+\frac{\left|B_{0 j}\right|^{2}}{\Delta_{k}^{\prime}}\right),
$$

and, finally, we also have

$$
\begin{aligned}
G_{y} & \equiv 1-\frac{\left(e^{2} \omega^{2}\left|B_{0 x}\right|\left|B_{0 y}\right|\right)^{2}}{2 \Delta_{x} \Delta_{y}\left(\Delta_{z}^{\prime}\right)^{2} F_{x} F_{y}}, \\
G_{z} & \equiv 1-\frac{\left(e^{2} \omega^{2}\left|B_{0 x}\right|\left|B_{0 z}\right|\right)^{2}}{4 \Delta_{x}\left(\Delta_{y}^{\prime}\right)^{2} \Delta_{z} F_{x} F_{z}} .
\end{aligned}
$$

We note that in all the susceptibilities above $\chi_{i j}=\chi_{j i}^{*}$ only when the field amplitudes are real and the optical frequency is far from any resonance.

In the unperturbed principal axis system, the susceptibility tensor has diagonal components $\chi_{11}(\omega), \chi_{22}(\omega)$, and $\chi_{33}(\omega)$, given by Eqs. (3.14), (3.17), and (3.20). Upon excitation with intense light off-diagonal terms are contributed in various orders as specified by Eqs. (3.8)-(3.28). In the present calculation, all such terms are magnetic in origin since quadratic and higher-order electric field terms have specifically been excluded. Note that the mixed character susceptibilities $\chi(0), \chi(\omega)$, and $\chi(2 \omega)$ can generate either electric or magnetic dipoles. ED fields appear at frequencies $0, \omega$, and $2 \omega$ as indicated by the argument of $\chi$. However, because the magnetic dipole moment oscillates at $\pm \omega$ in the rotating frame of $\mathrm{E}$ (see Section 2), the MD fields appear at $\omega$ in the lab frame. Considering response at frequency $\omega$ only, and specializing again to the case of a real $x$-polarized wave propagating along $z$, the susceptibility tensor far from any resonances can be taken to have the form

$$
\chi=\left[\begin{array}{ccc}
\chi_{11} & 0 & -i\left|\chi_{13}\right| \\
0 & \chi_{22} & 0 \\
i\left|\chi_{31}\right| & 0 & \chi_{33}
\end{array}\right]
$$

When the medium is lossless, the quantities $\left|\chi_{13}(2 \omega)\right|$ and $\left|\chi_{31}(2 \omega)\right|$ are real and equal. These off-diagonal terms form polarizations such as $P_{z}^{(2)}(2 \omega)=i \varepsilon_{0}\left|\chi_{31}^{(2)}(2 \omega)\right| E_{x}(\omega)$ that account for $z$-polarized magnetic emission at the fundamental frequency $\omega$. By determining the eigenvalues of the tensor $\chi$ in the presence of light the components of $\chi$ in the diagonalized (primed) reference frame are found to be $\chi_{11}^{\prime}=\chi_{11}-\left|\chi_{13}\right|, \chi_{22}^{\prime}=\chi_{22}$, and $\chi_{33}^{\prime}=\chi_{11}+\left|\chi_{13}\right|$. Noting that $\chi_{11}^{\prime} \neq \chi_{22}^{\prime} \neq \chi_{33}^{\prime}$ even if the material is initially isotropic $\left(\chi_{11}=\chi_{22}=\chi_{33}\right)$, we conclude that optical magnetization generates linear birefringence in the medium. Similarly, for probe waves that are not collinear with the incident field, it generates circular birefringence.

Self-induced magneto-optic birefringence is weak unless the cyclotron frequency approaches the optical frequency or large resonant enhancement is present. However if these conditions are met, wave energy will propagate in a direction that is slightly different from $\hat{z}$ in the laboratory frame, as one can easily show. By solving the wave equation $\bar{k} \times(\bar{k} \times \bar{E})+\left(\omega^{2} / c^{2}\right) \bar{E}=-\left(\omega^{2} / c^{2}\right) \overleftrightarrow{\chi} \bar{E}$, refractive indices for $\hat{x}$ - and $\hat{y}$-polarized light are found to be $n_{x}=\sqrt{1+\chi_{11}-\left(\left|\chi_{13}\right|^{2} /\left[1+\chi_{11}\right]\right)}$ and $n_{y}=\sqrt{1+\chi_{22}}$, respectively, and an axial electric field component develops with a relative amplitude given by

$$
E_{z}=\left(\frac{-\chi_{31}}{1+\chi_{11}}\right) E_{x}
$$

Hence there is a small angle between the Poynting vector and the optical wave vector $\bar{k}$ given by $\theta=\tan ^{-1}\left(\left|\chi_{13}\right| /\left[1+\chi_{11}\right]\right)$. This angular deviation of the Poynting vector results in a "walk-off" effect that is similar to that encountered in naturally birefringent crystals. Here, though, it results from magnetic anisotropy. The direction of $\bar{S}$ differs from $\hat{k}$ because $\bar{B}$ is not parallel to $\bar{H}$.

The off-diagonal susceptibilities derived above are nonlinear in their dependences on optical field amplitudes, even though quadratic or higher-order dependences in $E$ were omitted from the theoretical model. Because the definitions Eqs. (3.5)-(3.7) remove the linear electric field dependence from the calculated susceptibilities, any residual field dependence is fundamentally magnetic in origin. Although it is common practice to decompose susceptibilities using a power series to separate first-, second-, and higher-order dependences on $\bar{E}$, Eqs. (3.8)-(3.28) have the merit of revealing novel effects such as the selfinduced birefringence and beam walk-off effects described above that are the direct result of optical magnetization.

With these results in hand, an expression for the magnitude of the off-resonant optical magnetization $\bar{M}$ itself can be obtained. For a dielectric medium (with conduction current density $\bar{J}_{c}=0$ ), the relationship between $\bar{M}$ and $\bar{J}_{M}$ is expressible in terms of components parallel and perpendicular to the electric field: $\left|(\bar{\nabla} \times \bar{M})_{\perp}\right|$ 
$=\left|\left(\bar{J}_{M}\right)_{\perp}\right|=\left|\left(\bar{J}_{M}\right)_{\|}\right|=\left|(\bar{\nabla} \times \bar{M})_{\|}\right|$. As determined earlier, the magnetic current is also related to the motion of real charges by

$$
\left|\left(\bar{J}_{M}\right)_{\|}\right|=-R\left|\left(\bar{J}_{E}\right)_{\|}\right|=-R\left|\frac{\partial(\bar{P})_{\|}}{\partial t}\right| .
$$

Hence it is apparent that the magnitudes of magnetization and electric polarization are related by

$$
\left|(\bar{\nabla} \times \bar{M})_{\perp}\right|=\left|(\bar{\nabla} \times \bar{M})_{\|}\right|=-R\left|\frac{\partial(\bar{P})_{\|}}{\partial t}\right| .
$$

By evaluating the indicated derivatives for an $x$-polarized plane wave propagating in the $z$ direction and using the dispersion relation of light together with the constitutive relation $\bar{B}=\mu \bar{H}=\mu_{0}(\bar{H}+\bar{M})$, the optical magnetization per unit volume parallel to $\bar{B}=B(t) \hat{y}$ is found to be

$$
\bar{M}(t)=-c R P(t) \hat{y} .
$$

At high intensities, nonlinear restoring forces in real atomic systems eventually cause nonlinear optical effects to appear that are proportional to powers of the electric field ( $E^{2}, E^{3}$, etc.). Since the nonlinear magnetization in Eq. (3.36) scales in proportion to polarization at elevated intensities, one can surmise that intense, purely magnetic nonlinear effects may be observable at moderate intensities too. This is borne out by experimental results on magnetic continuum generation shown in Section 4. There, observations are presented of intense magnetic whitelight generation in two dielectric media and the magnetic emission is found to resemble the corresponding, highly nonlinear electric process closely.

An additional consequence of intense magnetic response at optical frequencies is that Poynting's theorem must be modified to include magnetic energy storage in the medium. Customarily [10] the rate of energy loss at an arbitrary point in space is equated to the negative divergence of the Poynting vector $S$. Including dynamic magnetization, this yields

$$
\begin{aligned}
-\bar{\nabla} \cdot \bar{S}= & \bar{E} \cdot \bar{J}+\frac{1}{2} \frac{\partial}{\partial t}\left(\mu_{0} \bar{H} \cdot \bar{H}+\varepsilon_{0} \bar{E} \cdot \bar{E}\right) \\
& +\left(\bar{H} \cdot \frac{\partial}{\partial t} \mu_{0} \bar{M}+\bar{E} \cdot \frac{\partial}{\partial t} \bar{P}\right) .
\end{aligned}
$$

The energy loss rate is determined by three terms on the right-hand side of Eq. (3.37). The first two are irreversible (ohmic) losses and changes in the energy stored in the free space electric and magnetic fields, respectively. The third and fourth terms describe changes in energy stored in the motion of charges. When the magnetization is no longer negligible $(\partial \bar{M} / \partial t=0)$ its effect on energy balance must be taken into account.

\section{EXPERIMENTS AND RESULTS}

As a preamble to the description of experimental procedures, we note how $\mathrm{ED}$ and $\mathrm{MD}$ scattered radiation are distinguishable on a purely experimental basis. In Fig. 4,

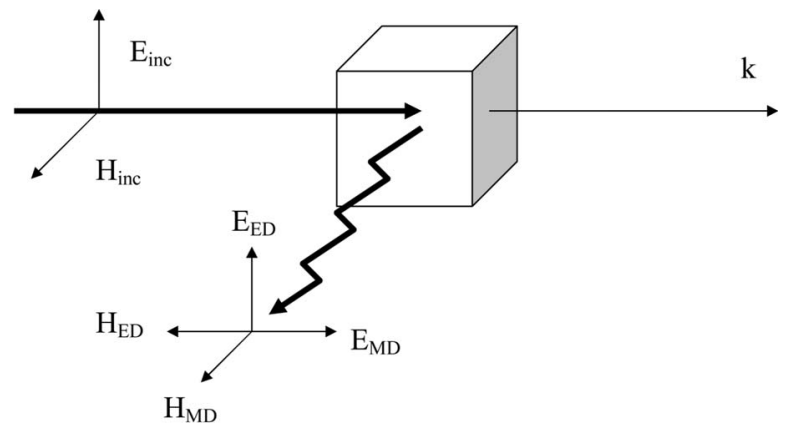

(a)

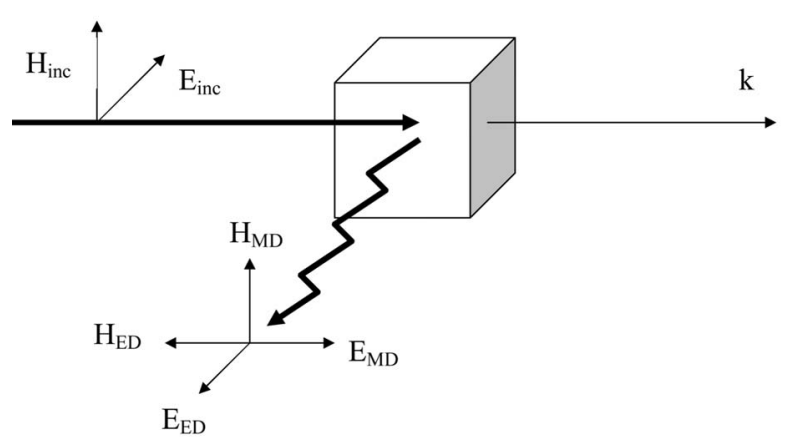

(b)

Fig. 4. Relative orientations of incident and scattered dipole electromagnetic fields for (a) vertical incident polarization (which yields a maximum intensity for ED radiation detected through a vertical analyzer and an MD null), and (b) horizontal incident polarization (which yields a maximum intensity for MD radiation detected through a horizontal analyzer and an ED null).

we illustrate how rotation of a signal polarizer can be used to separate linearly polarized ED and MD signals. When the incident polarization is vertical [Fig. 4(a)], the radiant MD magnetic field points in the direction of observation, producing a null intensity for $\mathrm{MD}$ radiation measured in this direction. When the incident polarization is horizontal [Fig. 4(b)], the radiant MD magnetic field is vertical, producing a maximum intensity in the direction of observation, consistent with Poynting's vector. For these same two polarizations the radiant ED fields produce a maximum and a null. Hence the incident polarizations that produce maximum ED and MD scattered intensities are orthogonal and the corresponding fields may be readily separated using vertical and horizontal analyzers in a standard $90^{\circ}$ scattering geometry.

Experiments were performed with three sources: A chirped pulse amplified laser system, a cw mode-locked Ti:sapphire oscillator and an Ar gas laser. The highest peak powers were obtained from a frequency-doubled Er:glass fiber laser that was regeneratively amplified in Ti:sapphire at a repetition rate of $1 \mathrm{kHz}$ (Clark MXR CPA2001). This yielded average output powers as high as $100 \mathrm{~mW}$ in pulse trains of $\sim 150 \mathrm{fs}$ pulses at a fixed wavelength of $775 \mathrm{~nm}$. To measure radiation patterns, low average powers in the neighborhood of $3.5 \mathrm{~mW}$ were focused with a $30 \mathrm{~cm}$ lens to avoid optical component damage and to limit peak intensities at the sample to $I<10^{13} \mathrm{~W} / \mathrm{cm}^{2}$. Power dependence was measured with collimated beams 
of diameter $<3 \mathrm{~mm}$ over the lowest intensity range that gave observable signals, to eliminate the possibility of polarization rotation of signals crossing the interaction region toward the detector. A cw mode-locked Ti:sapphire oscillator provided output pulses of $\sim 100$ fs duration centered at $810 \mathrm{~nm}$, and operated at a repetition rate of $80 \mathrm{MHz}$. By using a $2 \mathrm{~cm}$ focusing lens, peak intensities at the sample of up to $10^{10} \mathrm{~W} / \mathrm{cm}^{2}$ were obtainable with this source. Finally, output in the $1-10 \mathrm{~W}$ range was available from a cw Ar laser operating at $514 \mathrm{~nm}$. Peak intensity achieved in this case using a $20 \mathrm{~cm}$ focusing lens never exceeded $10^{5} \mathrm{~W} / \mathrm{cm}^{2}$.

Light from these sources was directed through a double Fresnel rhomb or a half-wave-plate into liquid samples of $\mathrm{CCl}_{4}, \mathrm{H}_{2} \mathrm{O}$, and $\mathrm{C}_{6} \mathrm{H}_{6}$. The intensity of light scattered at right angles, polarized either transverse (ED) or parallel (MD) to the incident wave vector, was measured through a calcite linear polarizer and $10 \mathrm{~nm}$ bandpass interference filter. In the case of the high power experiments, many wavelengths from the ultraviolet to the infrared region were selectable for signal detection, because of the broad spectral redistribution of light that accompanied whitelight generation. The mode-locked oscillator could not reach this threshold, however, even with tight focusing, so no such spectral reshaping took place in the samples. In this instance only Rayleigh scattering at the incident wavelength was generated and detected at $810 \mathrm{~nm}$. Detection wavelength in experiments with linearly polarized $\mathrm{Ar}$ laser radiation was $514 \mathrm{~nm}$.

Radiation patterns of light with ED or MD polarization were determined by recording intensity versus rotation angle of the input polarization [13]. Since only isotropic samples were studied, this accurately mapped out the spatial distribution of scattered intensity at each signal polarization. To establish the multipole character of the emission reliably on a purely experimental basis, measurements of this type were always performed with intensities at the sample of $I<10^{11} \mathrm{~W} / \mathrm{cm}^{2}$ to avoid unwanted laser-induced polarization distortions in beam control optics or samples.

In experiments with amplified pulses time-averaged signals were recorded with a Hamamatsu 636-10 photomultiplier and standard photon counting techniques. Linearly polarized beams of average input power $\sim 3 \mathrm{~mW}$ were softly focused into liquid samples of $\mathrm{CCl}_{4}, \mathrm{C}_{6} \mathrm{H}_{6}$, and $\mathrm{H}_{2} \mathrm{O}$ prefiltered through $0.2 \mu \mathrm{m}$ meshes. Detector sensitivity was found to vary by approximately $40 \%$ between orthogonal signal polarizations, so signal corrections were necessary. A precision right-angle prism was used during alignment to define the $90^{\circ}$ scattering angle within 3 arc min. Two optical stops of diameter $3 \mathrm{~mm}$ separated by $10 \mathrm{~cm}$ were placed between sample and detector to limit both the wave vector bandwidth and to maintain the relative contribution of out-of-plane electric dipole signal intensities below $10^{-4}$. $\mathrm{A} \mathrm{CaCO}_{3}$ analyzer with $10^{-4}$ rejection ratio was selected and carefully positioned after the apertures to eliminate motion of the signal beam on the photocathode at different orientations of the analyzer. Representative results from these experiments are shown in the polar plots of Figs. 5(a) and 5(b).

Experiments with the mode-locked oscillator, at greatly reduced peak intensities, utilized mechanical chopping of
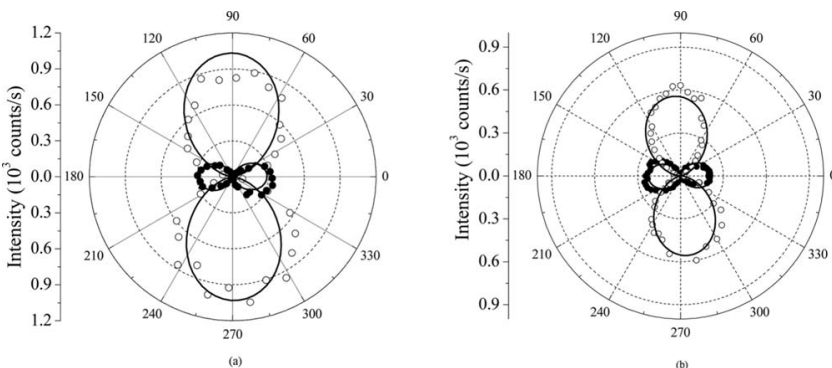

Fig. 5. Polar plots of experimental radiation patterns of magnetic (solid circles) and electric (open circles) scattering intensities obtained using amplified pulses above white-light threshold in (a) $\mathrm{CCl}_{4}$ and (b) $\mathrm{H}_{2} \mathrm{O}$.

the input beam and synchronous detection with a polarization-insensitive EG\&G FND-100 photodiode. Up to $400 \mathrm{~mW}$ of average power was focused at $50 \%$ duty cycle into the sample region with a $2.0 \mathrm{~cm}$ lens providing excellent signal levels through apertures reduced to $1 \mathrm{~mm}$, which kept spurious signal levels below $10^{-5}$. Radiation patterns obtained in this way at the central laser wavelength $810 \mathrm{~nm}$ for all three liquid samples are shown in Fig. 6. The solid curves are fits to dipolar $\cos ^{2} \phi$ and $\sin ^{2} \phi$ angular dependences. Identical experiments using approximately $1 \mathrm{~W}$ of output from an Ar laser light focused into $\mathrm{CCl}_{4}$ produced no measurable signal with axial polarization at all and will not be discussed further.

Peak intensities in the oscillator experiments were limited to $I \ll 2 \times 10^{10} \mathrm{~W} / \mathrm{cm}^{2}, 1000$ times below white-light and bubble formation thresholds in $\mathrm{H}_{2} \mathrm{O}$ [14]. However the focal spot position was still slightly intensitydependent due to incipient self-focusing, necessitating the use of an unfocused beam from the amplified source for reliable determinations of the intensity dependence of magnetic scattering (Fig. 7). These relative MD-ED measurements, at power levels intermediate between those of

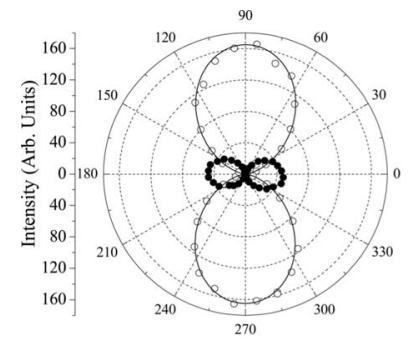

(a)

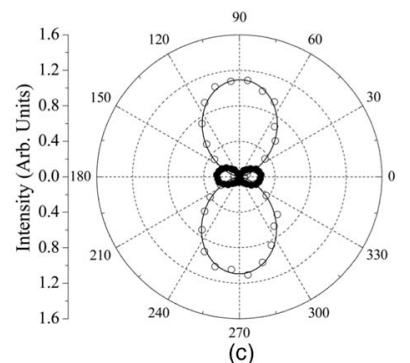

Fig. 6. Radiation patterns of MD (solid circles) and ED (open circles) scattering intensities obtained using unamplified pulses below white-light threshold: (a) $\mathrm{CCl}_{4}$, (b) magnified view of the magnetic component in $\mathrm{CCl}_{4}$, (c) $\mathrm{H}_{2} \mathrm{O}$, and (d) $\mathrm{C}_{6} \mathrm{H}_{6}$. 


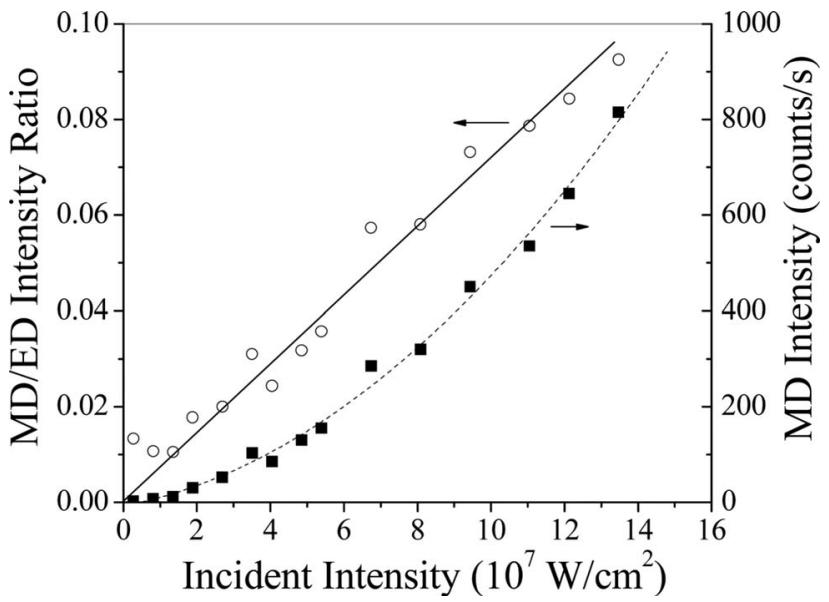

Fig. 7. Experimental intensity of magnetic dipole scattering versus input intensity in $\mathrm{CCl}_{4}$. The solid (dashed) curve is a linear (quadratic) regression through the data.

the Ar laser and white-light experiments, showed a transition from negligible magnetic scattering at low input power to intense signal levels at higher intensities.

\section{DISCUSSION}

Radiation patterns measured above white-light threshold revealed two-lobed patterns of scattered light intensity measured with the analyzer parallel (closed circles) or perpendicular to the optical wave vector (open circles) of the laser beam. The open circles in Fig. 5 correspond to familiar ED scattering. The closed circles correspond to magnetic emission, polarized orthogonal to the ED pattern. In Figs. 5(a) and 5(b) the scattered intensity ratios are $S_{M} / S_{E}=0.22 \pm 0.05$ and $S_{M} / S_{E}=0.38 \pm 0.10$, respectively. These results therefore agree quantitatively with the maximum ratio for MD scattering $S_{M} / S_{E}=0.25$ determined in Eq. (2.21), within experimental error. However, the effects of intensity fluctuations from the laser and the white-light generation process are evident in the data, and other measurements (discussed below) provided much clearer determinations of the multipole character of the magnetic radiation and its generation mechanism. Also, ionization is thought to accompany white-light generation [15]. Hence any interpretation of the experimental results in Figs. 5(a) and 5(b) as arising purely from bound electron dynamics is compromised. The remainder of this discussion therefore focuses on results from experiments in un-ionized dielectric samples.

Saturated magnetic scattering was observed at the fundamental wavelength of a $\mathrm{Ti}: \mathrm{Al}_{2} \mathrm{O}_{3}$ oscillator at intensities as low as $10^{10} \mathrm{~W} / \mathrm{cm}^{2}$ [Figs. $6(\mathrm{a})-6(\mathrm{~d})$ ], a result that is in quantitative accord with Eq. (2.10) assuming parametric resonance and a linewidth of $\gamma_{2}=2 \omega_{c} \approx 3.2$ $\times 10^{11} \mathrm{rad} / \mathrm{s}$ or less. Because of the absence of any depolarized background scattering in $\mathrm{CCl}_{4}$ and reduced amplitude fluctuations when a mode-locked oscillator was used, the measurements of Fig. 6(b) plainly showed that the radiation is purely magnetic dipole in character. This is compelling evidence of intense MD response from bound electrons in dielectric atomic systems at intensities of $\sim 10^{10} \mathrm{~W} / \mathrm{cm}^{2}[13]$. The ratios of MD-ED scattering inten- sity in Figs. 6(a), 6(c), and 6(d) are 0.27 $\pm 0.04,0.23 \pm 0.04$, and $0.28 \pm 0.04$, respectively; again in agreement with Eq. 2.26 within experimental error. On the other hand, the absence of magnetic response in experiments performed with the cw Ar laser indicated that no observable MD emission takes place at low intensities. Hence a transition between these two regimes must exist, as confirmed by the results of Fig. 7. The MD signals in Fig. 7 show a quadratic dependence on incident intensity and an accurately linear ratio of MD-ED signals, providing good agreement with the predicted power dependence and nonlinear mechanism of MD generation in Eq. (2.10) below saturation.

\section{CONCLUSIONS AND SUMMARY}

The main results of this paper are the observation and explanation of intense magnetic radiation induced by the passage of light through dielectric media: Magnetic continuum generation over a broad range of wavelengths and magnetic scattering at the fundamental wavelength. Experimentally, we have demonstrated that observed magnetic scattering is purely dipolar, is nonlinear in origin, and is comparable to ED scattering only at elevated (but nonrelativistic) intensities in bound electron systems by virtue of resonant enhancement. All the results of this paper (experimental and theoretical) are consistent with the dipole approximation $(a / \lambda \ll 1)$, in contradistinction to [16]. Our model explains the unprecedented intensity of MD emission as the result of parametrically enhanced magnetic forces acting on bound electrons, exceeding the standard limit of magnetic response expressed by Eq. (1.2) through a nonlinear mechanism whereby moderately intense optical fields induce large magnetic displacement currents. Many more magneto-optic effects analogous to known electro-optic effects may therefore be observable at lower intensities than previously thought.

Two such effects predicted in this paper are selfinduced linear birefringence and optically induced Faraday rotation. In the first, pump light produces fielddependent changes of the susceptibility elements $\left(\chi_{11}^{\prime}\right.$ $\left.\neq \chi_{22}^{\prime} \neq \chi_{33}^{\prime}\right)$ that affect pump propagation. In the second, the polarization of an orthogonal probe librates about the propagation axis at the optical frequency (Section 3). A third effect, predicted by Eqs. (3.8)-(3.13), is magnetoelectric charge separation and static voltage generation across dielectric samples in the direction of propagation of light. Finally, there is the possibility of attaining negative refractive indices in homogeneous dielectric media by exploiting dynamic magnetic response. As shown in Section 2 , electric and magnetic response in bound electron systems share a common resonance condition. Consequently, for a sufficiently sharp dispersion feature, negative permeability should be attainable at small blue detunings from the common resonance provided that point symmetry of the meeting is such that rotations about $y$ transform in the same way as displacements along $x$. Negative refractive index behavior may be realizable in homogeneous dielectric media, since both $\varepsilon$ and $\mu$ could be negative over the same range of frequencies simultaneously. Low-loss approaches to subwavelength imaging [17], 
magnetic mirrors [18], nanolithography and electromagnetic cloaking [19] may become practical.

\section{APPENDIX A}

Here we show explicitly that an arbitrary current source $\bar{J}_{\omega}$ includes a radiant magnetic dipole in the first term of the multipole expansion of the vector potential $\bar{A}_{\omega}$. After decomposing the current into components parallel and perpendicular to the optical electric field, the latter is shown to be the Cartesian projection of the curl of a dipolar magnetization from the solenoidal part of $\bar{J}_{\omega}$. If a (nonlinear) mechanism exists to generate a large magnetic source current of this type, magnetic dipole radiation that is not limited to a small fraction of the electric dipole radiation is generated.

In the presence of magnetic forces, there is no reason to assume that the vector potential is parallel to any particular incident field direction. Hence we write Eq. (1.1) as the sum of source terms parallel and perpendicular to the incident optical field $\bar{E}$.

$$
\frac{4 \pi R}{\mu_{0}} \bar{A}_{\omega}(\bar{r})=\int_{V} \bar{J}_{\|, \omega}\left(\bar{r}^{\prime}\right) \mathrm{d} V+\int_{V} \bar{J}_{\perp, \omega}\left(\bar{r}^{\prime}\right) \mathrm{d} V+\cdots
$$

(For the remainder of this development, the subscript indicating Fourier component $\omega$ is dropped.) The first term on the right of Eq. (A1) contains the usual electric dipole term generated by the electric field. The second term is typically assumed to be negligible in isotropic media at nonrelativistic intensities because the electric field only accelerates charges in the direction of $\bar{E}$ itself. However, if an open loop current component $\bar{J}_{\perp}$ with the property $\bar{\nabla} \cdot \bar{J}_{\perp}=0$ is generated by the optical $\bar{B}$ field by any means - for example, by parametric resonance between the motions driven by $\bar{E}$ and $\bar{B}$ in a bound electron system-the calculation below shows that magnetic dipole radiation contributes to $\bar{A}$ in first order.

The usual expression for the magnetic dipole moment $\bar{m}$ in a source volume $V$ is

$$
\bar{m}=\frac{1}{2} \int_{V}(\bar{r} \times \bar{J}) \mathrm{d} V
$$

Here $\bar{J}$ is implicitly azimuthal with respect to $\bar{B}$ (i.e., $\bar{J}$ $=\bar{J}_{M}=J_{M \theta} \hat{\theta}$ for propagation along $\hat{z}$ ). Unless $\bar{J}$ is azimuthal, according to Eq. (A2), it cannot contribute to the magnetic moment. The differential element of $\bar{m}$ is then related to the magnetization per unit volume $\bar{M}$ by $d \bar{m}$ $=\bar{M} \mathrm{~d} V$. Consequently $\bar{M}=\frac{1}{2}\left[\bar{r} \times \bar{J}_{M}\right]$, and

$$
\bar{\nabla} \times \bar{M}=\bar{\nabla} \times\left[\frac{1}{2}\left(\bar{r} \times \bar{J}_{M}\right)\right] .
$$

Cartesian components of the volume integral of Eq. (A3) are

$$
\left[\int_{V}[\bar{\nabla} \times \bar{M}] \mathrm{d} V\right]_{i}=\frac{1}{2} \int_{V} \varepsilon_{i j k} \frac{\partial}{\partial x_{j}} \varepsilon_{k l m} x_{l}\left(2 J_{M \theta}\right)_{m} \mathrm{~d} V
$$

where the indices $i, j, k$, and $m$ may be $x, y$, or $z$. The Levi-Civita symbol $\varepsilon_{i j k}$ is nonzero for unrepeated indices, has the value +1 if the indices form a cyclic permutation of $x y z$, and is -1 if the indices are anticyclic. The factor of 2 in the integrand accounts for the projection of the oscillatory circular current $\bar{J}_{M}$ onto Cartesian axes [see Eq. (2.8)]. Equation (A4) simplifies to

$$
\left[\int_{V}[\bar{\nabla} \times \bar{M}] \mathrm{d} V\right]_{i}=\varepsilon_{i j k} \varepsilon_{l m k} \int_{V} \frac{\partial}{\partial x_{j}} x_{l}\left(J_{M \theta}\right)_{m} \mathrm{~d} V,
$$

and can be re-expressed in terms of Kronecker delta symbols using the identity $\varepsilon_{i j k} \varepsilon_{l m k}=\delta_{i l} \delta_{j m}-\delta_{i m} \delta_{j l}$ to obtain [20]

$$
\begin{aligned}
{\left[\int_{V}[\bar{\nabla} \times \bar{M}] \mathrm{d} V\right]_{i} } & =\left(\delta_{i l} \delta_{j m}-\delta_{i m} \delta_{j l}\right) \int_{V} \frac{\partial}{\partial x_{j}} x_{l}\left(J_{M \theta}\right)_{m} \mathrm{~d} V, \\
& =\int_{V}\left[\frac{\partial}{\partial x_{j}} x_{l}\left(J_{M \theta}\right)_{j}-\frac{\partial}{\partial x_{j}} x_{j}\left(J_{M \theta}\right)_{i}\right] \mathrm{d} V .
\end{aligned}
$$

Using the product rule to expand the derivatives in Eq. (A6), together with the identity $[\bar{x} \cdot \bar{\nabla}]\left(J_{M \theta}\right)_{i}=\bar{\nabla} \cdot\left[\bar{x}\left(J_{M \theta}\right)_{i}\right]-\left(J_{M \theta}\right)_{i}[\bar{\nabla} \cdot \bar{x}]=\bar{\nabla} \cdot\left[\bar{x}\left(J_{M \theta}\right)_{i}\right]-3\left(J_{M \theta}\right)_{i}$, Eq. (A6) becomes

$$
\begin{aligned}
{\left[\int_{V}[\bar{\nabla} \times \bar{M}] \mathrm{d} V\right]_{i} } & =\int_{V}\left[\left(\bar{\nabla} \cdot \bar{J}_{M}\right) x_{i}+\left(J_{M \theta}\right)_{i}\right. \\
& \left.-\bar{\nabla} \cdot\left[\bar{x}\left(J_{M \theta}\right)_{i}\right]\right] \mathrm{d} V .
\end{aligned}
$$

Because there are no magnetic charges, magnetic currents are divergence-free $\left(\bar{\nabla} \cdot \bar{J}_{M}=0\right)$. Consequently the first term on the right of Eq. (A7) vanishes, yielding

$$
\left[\int_{V}[\bar{\nabla} \times \bar{M}] \mathrm{d} V\right]_{i}=\int_{V}\left[\left(J_{M \theta}\right)_{i}-\bar{\nabla} \cdot\left[\bar{x}\left(J_{M \theta}\right)_{i}\right]\right] \mathrm{d} V .
$$

In a homogeneous medium where the charge response is uniform throughout space, the current amplitude $J_{M \theta}$ is also a constant that can be removed from the second integral on the right. Using the divergence theorem to rewrite this last term as an integral over the surface $S$ that encloses source volume $V$, we find that

$$
\int_{V}\left[\bar{x}\left(J_{M \theta}\right)_{i}\right] \mathrm{d} V=\left(J_{M \theta}\right)_{i} \int_{S} \bar{x} \mathrm{~d} s=0,
$$

since $\bar{x}$ is positive as much as it is negative over the surface $S$. Hence

$$
\left[\int_{V}[\bar{\nabla} \times \bar{M}] \mathrm{d} V\right]_{i}=\int_{V}\left(J_{M \theta}\right)_{i} \mathrm{~d} V .
$$


Thus circular current components with the property $\bar{\nabla} \cdot \bar{J}=0$ can produce magnetization (and magnetic dipole radiation) via the first term in the multipole expansion. Equation (A10) is valid regardless of the magnitude of $\partial \bar{P} / \partial t$, and applies to insulators or conductors alike (see below). Consequently, dynamic magnetic moments can be much larger than previously thought at optical frequencies. In Eq. (3.36) the magnetization was shown to be proportional to the electric polarization in insulators, and was not limited in magnitude to a small fraction of the order of $(1 / 137)^{2}$ times the electric dipole moment. This applies not only to dielectrics but also to weakly conductive media, where $\bar{\nabla} \times \bar{M}$ may be comparable to $\partial \bar{P} / \partial t$ while still consistently representing the curl of a magnetic dipole moment per unit volume, as we now show.

If the total current ( $\left.\bar{J}_{\text {TOT }}\right)$ is simply decomposed into an electrical (nonsolenoidal) part governed by the usual charge continuity equation and a magnetic (solenoidal) part with zero divergence then, provided $\chi_{m} \ll 1$ as in the approximation of Section 2, one finds from an integration of Ampère's law [6] that

$$
0=\bar{J}_{\text {sol }}+\bar{J}_{\text {nonsol }}+\bar{\nabla} \times \bar{M}+\partial \bar{P} / \partial t,
$$

where $\bar{J}_{\mathrm{TOT}}=\bar{J}_{\mathrm{sol}}+\bar{J}_{\text {nonsol }}$. Equation (A11) may be decomposed into two distinct parts:

$$
\bar{J}_{\mathrm{sol}}+\bar{\nabla} \times \bar{M}=0
$$

where

$$
\bar{\nabla} \cdot \bar{J}_{\mathrm{sol}}=0,
$$

and

$$
\bar{J}_{\text {nonsol }}+\partial \bar{P} / \partial t=0
$$

where

$$
\bar{\nabla} \cdot \bar{J}_{\text {nonsol }} \neq 0 \text {. }
$$

This decomposition in terms of physically distinguishable portions of the total current eliminates any restriction on $\bar{\nabla} \times \bar{M}$ due to polarization [7], since $\bar{P}$ does not appear in Eq. (A12). Thus, there is no inherent restriction on $|\bar{M}|$ at high frequencies other than that imposed by the simple fact that of all the charges within a volume that can be set in motion by the time-varying electric component of an electromagnetic wave, at most half can be deflected around the origin to form a positive magnetic current by the magnetic component [13].

\section{ACKNOWLEDGMENTS}

The authors gratefully acknowledge early discussions with N. Sharma, technical assistance by J. Whitaker, critical review of a draft manuscript by $\mathrm{H}$. Winful, and funding by the National Science Foundation (DMR0502715 and CISE-0531086) and the U.S. Air Force Office of Scientific Research (AFOSR) (F49620-03-10389). S. C. Rand also thanks several individuals and institutions for their hospitality during portions of this research: C. B. de Araujo at Universidade Federal de Pernambuco, O. Svelto at Politecnico di Milano, W. Sibbett at the University of St. Andrews, and C. Mirasso at the Universitat de les illes Balears.

\section{REFERENCES}

1. L. P. Pitaevskii, "Electric forces in a transparent dispersive medium," Sov. Phys. JETP 12, 1008-1013 (1961).

2. Y. R. Shen and N. Bloembergen, "Interaction between light waves and spin waves," Phys. Rev. 143, 372-384 (1966).

3. P. S. Pershan, J. P. Van der Ziel, and L. D. Malmstrom, "Theoretical discussion of the inverse Faraday effect, Raman scattering, and related phenomena," Phys. Rev. 143, 574-583 (1966).

4. A. V. Kimel, A. Kirilyuk, F. Hansteen, R. V. Pisarev, and T. Rasing, "Non-thermal optical control of magnetism and ultrafast laser-induced spin dynamics in solids," J. Phys.: Condens. Matter 19, 043201 (2007).

5. G. A. Mourou, C. P. J. Barty, and M. D. Perry, "Ultrahighintensity lasers: Physics of the extreme on a tabletop," Phys. Today 51, 22-28 (1998).

6. W. K. H. Panofsky and M. Phillips, Classical Electricity and Magnetism, 2nd ed. (Addison-Wesley, 1962), p. 132.

7. L. Landau and E. M. Lifshitz, Electrodynamics of Continuous Media, 2nd ed. (Pergamon, 1984), pp. 268-269.

8. See, for example, R. W. Boyd, Nonlinear Optics (Academic, 1992).

9. W. M. Fisher and S. C. Rand, "Dependence of optical magnetic response on molecular electronic structure," J. Lumin (to be published).

10. J. D. Jackson, Classical Electrodynamics, 2nd ed. (Wiley \& Sons, 1975)

11. W. K. H. Panofsky and M. Phillips, Classical Electricity and Magnetism, 2nd ed. (Addison-Wesley, 1962), pp. 245-248.

12. See, for example, A. H. Nayfeh and D. T. Mook, Nonlinear Oscillations (Wiley, 1979).

13. S. L. Oliveira and S. C. Rand, "Intense nonlinear magnetic dipole radiation at optical frequencies: Molecular scattering in a dielectric liquid," Phys. Rev. Lett. 98, 093901 (2007).

14. F. Courvoisier, V. Boutou, C. Favre, S. C. Hill, and J.-P. Wolf, "Plasma formation dynamics within a water microdroplet on femtosecond time scales," Opt. Lett. 28, 206-208 (2003).

15. A. Brodeur and S. L. Chin, "Ultrafast white-light continuum generation and self-focusing in transparent condensed media," J. Opt. Soc. Am. B 16, 637-650 (1999).

16. N. L. Sharma, "Nondipole scattering from liquids and nanoparticles,” Phys. Rev. Lett. 98, 217402 (2007).

17. J. B. Pendry, "Negative refraction makes a perfect lens," Phys. Rev. Lett. 85, 3966-3969 (2000).

18. D. Sievenpiper, L. Zhang, R. F. J. Broas, N G. Alexopolous, and E. Yablonovitch, "High-impedance, electromagnetic surfaces with a forbidden frequency band," IEEE Trans. Microwave Theory Tech. 47, 2059-2074 (1999).

19. J. B. Pendry, D. Schurig, and D. R. Smith, "Controlling electromagnetic fields," Science 312, 1780-1782 (2006).

20. See, for example, G. Arfken, Mathematical Methods for Physicists, 2nd ed. (Academic, 1970), p. 136. 